\title{
A Evolução do Cenário da Cardiogeriatria no Brasil: Novos Desafios para um Novo Mundo
}

\author{
The Evolving Landscape of the Geriatric Cardiology Field in Brazil: New Challenges for a New World \\ Caio de Assis Moura Tavares, ${ }^{10}$ André Feitosa Wanderley Cavalcanti, ${ }^{1}$ Wilson Jacob Filho ${ }^{1}$ \\ Unidade de Cardiogeriatria - Instituto do Coração (InCor) - Hospital das Clínicas HCFMUSP - Faculdade de Medicina - Universidade de São \\ Paulo, ${ }^{1}$ São Paulo, SP - Brasil
}

A necessidade de nos preparamos para o atendimento da população geriátrica já é reconhecida mundialmente há duas décadas. "Nós exercemos cardiologia geriátrica?". Assim o Dr William W. Parmley intitulou seu editorial de 1997, ${ }^{1}$ publicado no Journal of the American College of Cardiology (JACC), e considerado, por boa parte dos norte-americanos, como o ponto de partida para o desenvolvimento da Cardiogeriatria naquele continente. Apesar da existência, desde 1986, de uma sociedade (Society of Geriatric Cardiology - SGC), esta não atuava sob a tutela de nenhuma das duas grandes associações de Cardiologia americanas e carecia de maior relevância. Com o envelhecimento populacional, fenômeno mundial, o encontro do cardiologista com pacientes cada vez mais longevos havia se tornado frequente. A partir desse alerta, desenvolveram-se iniciativas para suprir a nova demanda. Um currículo online de educação continuada, elaborado entre a American College of Cardiology (ACC) e a SGC foi disponibilizado, em 2007, aos seus membros e divulgado em novo editorial, de 2008, do JACC. ${ }^{2}$ Em 2011, a SGC foi extinta e incorporada como Seção-Membro de Cardiologia Geriátrica da ACC.

Cabe ressaltar que, naquele período, o mundo já reconhecera a inexorabilidade do envelhecimento populacional e já percebera a importância de dotar os profissionais de uma capacitação superespecializada (a especialidade na faixa etária) e de dar a essa população cada vez mais predisposta a envelhecer, a possibilidade de ter suas demandas atendidas, conforme suas características individuais, mais do que pelas doenças que a acometiam.

No Brasil, o pioneirismo do Prof. Dr. Luís Gastão Costa Carvalho do Serro Azul, em 1982 (quatro anos antes da fundação da sociedade americana sobre o tema), iniciava a Unidade Clínica de Cardiogeriatria do Instituto do Coração (InCor), um movimento que colocou o nosso país em uma

\section{Palavras-chave}

Geriatria/tendências; Avaliação Geriátrica; Dinâmica Populacional; Cardiologia/tendências; Idoso; Assistência à Saúde; Idoso Fragilizado; Saúde para Idosos.

Correspondência: Caio de Assis Moura Tavares •

Unidade de Cardiogeriatria do Instituto do Coração do Hospital das Clínicas da Faculdade de Medicina da Universidade de São Paulo, Av. Dr Enéas de

Carvalho Aguiar, 44, 2o andar, sala 06. CEP 05403-900, São Paulo, SP - Brasil

E-mail: caio.tavares@usp.br

Artigo recebido em 20/05/2019, revisado em 20/08/2019,

aceito em 10/09/2019 posição de vanguarda na discussão desse tema. Na década de 90, a Cardiogeriatria foi reconhecida pela Sociedade Brasileira de Cardiologia, inicialmente, como um grupo de estudos em Cardiogeriatria (GEBRAC) e, desde 2005, como um departamento (DECAGE). Em 2006 e em 2014, dois artigos reforçaram para a sociedade médica Brasileira a importância da Cardiogeriatria - o primeiro, assinado pelo Prof. Dr Maurício Wajngarten, ${ }^{3}$ elencou os desafios que estavam por vir e a necessidade de preparação para atendimento dessa população idosa; o segundo, assinado pelo Prof. Roberto Franken e pelo Dr. Ronaldo Fernandes Rosa ${ }^{4}$ destacou o progresso pela criação do DECAGE para formação de treinamento em Cardiogeriatria, parcerias com a ACC, avanços em evidência científica gerada pelo departamento, bem como elencou habilidades essenciais para o atendimento pleno do idoso. Em conformidade com essa necessidade, a Sociedade Brasileira de Cardiologia publicou diretrizes específicas de Cardiogeriatria: a primeira em 2002; a segunda, em 2010, ${ }^{6}$ com sua atualização em 2019, ${ }^{7}$ além de elencar, na I Diretriz sobre Processos e Competências para a Formação em Cardiologia no Brasil, ${ }^{8}$ o conteúdo necessário aos profissionais em formação para o devido atendimento de doenças cardiovasculares no paciente idoso.

\section{A Essência da Cardiogeriatria}

Por tratar-se de advento recente e ainda não totalmente difundido, muitas vezes confunde-se a Cardiogeriatria com uma Geriatria praticada pelo cardiologista ou, simplesmente, como a Cardiologia aplicada ao paciente idoso, o que, apesar de constituir parte do âmago dessa disciplina, não representa a sua totalidade. Mais adequado seria defini-la como a integração do cuidado cardiovascular com uma abordagem adequada à idade e centrada no paciente ${ }^{9}$ e na sua funcionalidade - conceito já exposto previamente por nossos colegas ${ }^{3,4}$, mas que, com o passar dos anos, está tomando forma, com o uso de ferramentas específicas e pré-determinadas.

De modo objetivo, pode-se considerá-la como uma prática cardiológica, integrada aos $5 \mathrm{Ms}$ da Geriatria: medication (foco na prescrição do absolutamente necessário, visando restringir a polifarmácia, reduzir interações e efeitos adversos, respeitando os critérios de Beers na seleção de medicações adequadas ao idoso), mentation (vigilância, prevenção e tratamento dos distúrbios cognitivos), mobility (valorização e implementação de estratégias que visem manter a funcionalidade mecânica do paciente), multimorbidity

DOI: https://doi.org/10.36660/abc.20190292 


\section{Ponto de Vista}

(abordagem do paciente não com o olhar apenas sobre o sistema cardiovascular, mas considerando-se que a ocorrência de múltiplas comorbidades é a regra, e não a exceção, nesses indivíduos) e, por último, e literalmente mais importante, matters most (sempre valorizar a opinião do paciente sobre os benefícios e fardos que o tratamento irá acarretar, sob a ótica da sua biografia e valores pessoais, trazendo-o para o centro das decisões). Acrescentaríamos, ainda, um sexto e último $\mathrm{M}$, de multidisciplinaridade, pois o cuidado desses idosos deve estar coordenado de forma horizontal por um profissional, mas nunca isolado em sua figura, sendo importante a participação de outros especialistas e profissionais de saúde. Na Unidade de Cardiogeriatria do InCor, para todos os pacientes é realizada uma avaliação geriátrica ampla com o auxílio da ferramenta TAGA-10 (Targeted Geriatric asssesment) ${ }^{10,11}$

\section{A Evolução da Cardiogeriatria}

Com o passar dos anos, os avanços médicos, científicos e das condições de vida fizeram com que esses indivíduos não somente chegassem em grande número a idades avançadas, mas também com condições físicas e expectativas para o futuro que excedem o que se observava no passado, mudando os conceitos do que seria "ficar velho"12. Observamos ainda que, salvo poucas exceções, as doenças cardiovasculares são doenças do envelhecimento. No século XVII, o Dr Thomas Sydenham já havia declarado que "um homem é tão velho quanto suas artérias". De modo que o paciente típico do atendimento cardiológico é uma pessoa mais velha que carrega consigo, além de mais esperanças e anseios, também um número cada vez maior de comorbidades e deteriorações relacionadas ao envelhecimento que complicam o gerenciamento tradicional baseado em diretrizes ${ }^{13}$. Sabe-se, ainda, que o envelhecimento dá ao aparelho cardiovascular características diversas da juventude ${ }^{14-16}$, tornando ainda mais peculiar o atendimento do idoso.

O dogma tradicional de que "depois de certa idade, o paciente é velho demais para realizar procedimentos cardíacos invasivos" não tem espaço no cenário atual. Notadamente, com o avanço tecnológico vimos juntar-se ao arsenal terapêutico em Cardiologia opções que permitiram avançar no cuidado de afecções cardiovasculares que acometem desproporcionalmente indivíduos idosos - o surgimento dos Anticoagulantes Orais Diretos, Ressincronização Cardíaca, Dispositivos de Assistência Ventricular Esquerda, TAVI e Clipes Mitrais abrem novos horizontes terapêuticos. Paradoxalmente, o risco das intervenções (clínicas ou cirúrgicas) permanece alto nos muito idosos, trazendo a avaliação geriátrica e da fragilidade como uma ferramenta útil e necessária para a decisão terapêutica, podendo inclusive identificar indivíduos que se beneficiam do procedimento e aqueles nos quais o procedimento é fútil $^{9,17}$.

Vivemos na era da multimorbidade: dados do Medicare mostram que, entre seus usuários, ela ocorre em $63 \%$ dos que têm entre 65 e 75 anos, progredindo com a idade, chegando a ocorrer em $83 \%$ dos usuários acima de 85 anos ${ }^{18}$. Seu impacto econômico é igualmente impressionante, pois apenas 14\% dos beneficiários (os que relatam 6 ou mais condições crônicas) consomem 46\% do orçamento anual do programa (mais de U\$ 500 bilhões) ${ }^{13}$.
Os paradigmas atuais de tratamento das doenças cardiovasculares são limitados para esses pacientes. A abordagem atual para o atendimento do cardiologista é amplamente impulsionada por diretrizes de prática clínica de doença única - baseadas largamente em Ensaios Clínicos Randomizados que costumam excluir deliberadamente e sistematicamente idosos com multimorbidade: avaliam, predominantemente, desfechos duros e não consideram preservação física, cognição ou qualidade de vida associada à saúde em suas análises, que seriam muito mais relevantes na avaliação do paciente, nas últimas décadas ou anos de vida. Outra limitação para a aplicação dessas diretrizes, é que o enfoque na doença pode causar inadvertidamente efeitos prejudiciais no contexto da multimorbidade - a complexidade desse tema é tanto que, frequentemente, um tratamento acarreta o surgimento de uma nova doença ou descompensação de outra condição pré-existente ${ }^{19}$.

O conceito do fenômeno das "Sliding Doors" ${ }^{20}$ foi proposto para descrever como, no modelo atual de atendimento, um paciente com múltiplas comorbidades tem desfechos diferentes, a depender da porta em que ele entra primeiro. Por exemplo, um paciente apresentando um câncer colorretal oculto e doença arterial coronária, ao procurar primeiro o oncologista, tem diagnosticada a neoplasia, realiza cirurgia/ quimioterapia e durante o tratamento desenvolve insuficiência cardíaca; ao procurar primeiro o cardiologista, o mesmo paciente tem diagnosticada obstrução coronariana grave, é submetido à angioplastia, com uso de dupla antiagregação plaquetária e, após alguns meses, apresenta uma hemorragia digestiva significativa, sendo diagnosticado o câncer, já em fase mais avançada. Acreditamos, assim como Forman DE ${ }^{19}$, em um novo modelo, no qual o idoso com multimorbidades tenha seu cuidado centrado em um profissional com olhar geriátrico, que coordene de maneira horizontal os cuidados, com especialistas atuando pontualmente e sob comunicação, preferencialmente, com prontuários eletrônicos compartilhados. Em tal modelo, o paciente hipotético teria as duas doenças avaliadas e tratadas em momento oportuno.

Trata-se, pois, de um novo olhar para as enfermidades, tendo o paciente como foco principal, não apenas nos seus múltiplos componentes biológicos, mas também no seu universo biográfico, o que o transforma em único, mas nem por isso, excluído dos benefícios dos avanços tecnológicos que se mostraram eficazes em outras faixas etárias e que foram testados e comprovados também nessa fase avançada da vida.

Visto que nos foi dada a inestimável oportunidade de viver mais, seja esta também uma boa opção para viver melhor.

\section{Conclusão}

A Cardiogeriatria é um campo em evolução, ainda em processo de formação de sua identidade e definição do treinamento fundamental e mandatório. Em apenas duas décadas, evoluímos muito: identificamos gaps no conhecimento do idoso, demos os primeiros passos em direção ao estabelecimento de um currículo em cardiogeriatria, bem como desenvolvemos ferramentas específicas para a avaliação do idoso com doença cardiovascular - passos iniciais para uma subespecilidade ainda em formação. Programas de formação 
clínica na área ainda são raros - ao nosso conhecimento, na América do Norte, apenas na New York University, Vanderbilt University, University of Pittsburgh - nos Estados Unidos - e McGill University - no Canadá. No Brasil, existem no InCor, Instituto Dante Pazzanese e Escola Paulista de Medicina, abertos a Cardiologistas e Geriatras. Felizmente, esse ano, temos 6 profissionais em treinamento na nossa instituição maior número desde a abertura do programa. Que seja uma representação da evolução da cardiogeriatria e um incentivo para a longa jornada que ainda temos a frente, afinal os desafios não são poucos: i) estreitar os gaps de conhecimento para os idosos; ii) ampliar a participação dos idosos incluídos nos estudos clínicos; iii) avaliar desfechos que sejam relevantes para o paciente - cognição e qualidade de vida; iv) ampliar a capacidade de formar profissionais com treinamento específico em cardiogeriatria.

\section{Contribuição dos autores}

Concepção e desenho da pesquisa e Revisão crítica do manuscrito quanto ao conteúdo intelectual importante:
Tavares CAM, Cavalcanti AFW, Jacob Filho W; Redação do manuscrito: Tavares CAM, Cavalcanti AFW.

\section{Potencial conflito de interesses}

Declaro não haver conflito de interesses pertinentes.

\section{Fontes de financiamento}

O presente estudo não teve fontes de financiamento externas.

\section{Vinculação acadêmica}

Não há vinculação deste estudo a programas de pós-graduação.

\section{Aprovação ética e consentimento informado}

Este artigo não contém estudos com humanos ou animais realizados por nenhum dos autores.

\section{Referências}

1. Parmley WW. Do we practice geriatric cardiology? J Am Coll Cardiol. $1997 ; 29(1): 217-8$

2. Dove JT, Zieman SJ, Alexander K, Miller A. President's page: Cardiovascular care in older adults: the ACC and SGC partnership builds new curriculum. J Am Coll Cardiol. 2008;51(6):672-3.

3. Wajngarten M. Geriatric cardiology: a subspecialty or a need? Arq Bras Cardiol. 2006;87(3):e8-9.

4. Franken RA, Rosa RF. Cardiogeriatric, the future's cardiology? Arq Bras Cardiol. 2014;103(4):e52-4.

5. Gravina CF, Batlouni M, Sousa JEMR, Wajngarten M, Feitoso GS, Franken RA I Diretrizes do Grupo de Estudos em Cardiogeriatria da Sociedade Brasileira de Cardiologia. Arq Bras Cardiol. 2002;79(Supl 1):3-46.

6. Gravina CF, Franken R, Wenger N, Freitas EV, Batlouni M, Rich M, et al. [II Guidelines of Brazilian Society of Cardiology in geriatric cardiology]. Arq Bras Cardiol. 2010;95(3 Suppl 2):e16-76.

7. Feitosa-Filho GS, Peixoto JM, Pinheiro JES, Afiune Neto A, Albuquerque ALT, Cattani AC, et al. Updated Geriatric Cardiology Guidelines of the Brazilian Society of Cardiology - 2019. Arq Bras Cardiol. 2019;112(5):649-705.

8. Sociedade Brasileira de C, Sousa MR, Feitosa GS, Paola AA, Schneider JC, Feitosa-Filho GS, et al. [First guidelines of the Brazilian Society of Cardiology on processes and skills for education in cardiology in Brazil]. Arq Bras Cardiol. 2011;96(5 Suppl 1):4-24.

9. Bell SP, Orr NM, Dodson JA, Rich MW, Wenger NK, Blum K, et al. What to Expect From the Evolving Field of Geriatric Cardiology. J Am Coll Cardiol. 2015;66(11):1286-99.

10. Aliberti MJR, Covinsky KE, Apolinario D, Lee SJ, Fortes-Filho SQ, Melo JA, et al. A 10-min Targeted Geriatric Assessment Predicts Mortality in Fast-Paced Acute Care Settings: A Prospective Cohort Study. J Nutr Health Aging. 2019;23(3):286-90
11. Aliberti MJR, Apolinario D, Suemoto CK, Melo JA, Fortes-Filho SQ, Saraiva MD, et al. Targeted Geriatric Assessment for Fast-Paced Healthcare Settings: Development, Validity, and Reliability. J Am Geriatr Soc. 2018;66(4):748-54

12. Maurer MS. Age: a nonmodifiable risk factor? J Am Coll Cardiol $2003 ; 42(8): 1427-8$

13. Forman DE, Rich MW, Alexander KP, Zieman S, Maurer MS, Najjar SS, et al Cardiac care for older adults. Time for a new paradigm. J Am Coll Cardiol. 2011;57(18):1801-10.

14. Madhavan MV, Gersh BJ, Alexander KP, Granger CB, Stone GW. Coronary Artery Disease in Patients $>/=80$ Years of Age. J Am Coll Cardiol. 2018;71(18):2015-40.

15. Curtis AB, Karki R, Hattoum A, Sharma UC. Arrhythmias in Patients $>/=80$ Years of Age: Pathophysiology, Management, and Outcomes. J Am Coll Cardiol. 2018;71(18):2041-57.

16. Kodali SK, Velagapudi P, Hahn RT, Abbott D, Leon MB. Valvular Heart Disease in Patients $>/=80$ Years of Age. J Am Coll Cardiol. 2018;71(18):2058-72.

17. Afilalo J, Lauck S, Kim DH, Lefevre T, Piazza N, Lachapelle K, et al. Frailty in Older Adults Undergoing Aortic Valve Replacement: The FRAILTY-AVR Study. J Am Coll Cardiol. 2017;70(6):689-700.

18. Royal College of General Practioners. New Report highlights gaps in healthcare for increasing number of patients living with multiple conditions publication date: 04 November 2016. [Cited in 2018 Feb 10]. [Available from: https://www.rcgp.org.uk/about-us/news/2016/november/newreport-shows-gaps-in-healthcare-for-increasing-number-of-patients-withmultiple-conditions.aspx.

19. Forman DE, Maurer MS, Boyd C, Brindis R, Salive ME, Horne FM, et al. Multimorbidity in Older Adults With Cardiovascular Disease. J Am Coll Cardiol. 2018;71(19):2149-61.

20. Albini A, Pennesi G, Donatelli F, Cammarota R, De Flora S, Noonan DM Cardiotoxicity of anticancer drugs: the need for cardio-oncology and cardiooncological prevention. J Natl Cancer Inst. 2010;102(1):14-25. 